\title{
Solitons and Heat Transfer in Nonlinear Lattices with Cubic On-Site and Quartic Interaction Potentials
}

\author{
Rovinita Perseus, M. M. Latha \\ Department of Physics, Women's Christian College, Nagercoil, India \\ Email: lathaisaac@yahoo.com
}

Received 2 July 2014; revised 8 August 2014; accepted 18 September 2014

Copyright (C) 2014 by authors and OALib.

This work is licensed under the Creative Commons Attribution International License (CC BY). http://creativecommons.org/licenses/by/4.0/

(c) $\underset{\mathrm{EY}}{\mathrm{EY}}$ Open Access

\begin{abstract}
This paper deals with the transfer of soliton-like heat waves in nonlinear lattices with cubic onsite potential and quartic interparticle interaction potential. A model Hamiltonian was proposed using the second quantized operators and the same was averaged using a suitable wavefunction. The equations were derived numerically in the discrete form for the field amplitude. Moreover the resulting equations were analyzed analytically using the continuous approximation technique and the properties of heat transfer were examined theoretically.
\end{abstract}

\section{Keywords}

\section{Soliton, Nonlinear Lattice, Inhomogeneity}

Subject Areas: Dynamical System, Theoretical Physics

\section{Introduction}

The control of heat flow in electronic devices has drawn a great deal of attention in recent years since the rapid increase in temperature could detoriate the device in process of time [1]-[8]. Attempts have been made by scientists to study the transfer of heat by considering simple linear lattice models fixed with heat baths [9]-[12]. Later on these linear models have been extended to the nonlinear models of one, two and three dimensions [13]-[20]. Various models like thermal rectifiers, diodes and transistors have been designed using the nonlinearity property which has been found useful in controlling heat flow [21]-[25]. It is worth noting that the propagation of heat in the form of solitons has been confirmed by Toda and the concept of soliton has been established as a mode of heat propagation in nonlinear lattices [26]. Moreover all the broad ranging reviews of mathematical techniques and physical applications which have been performed previously are based on classical theories. The classical 
molecular dynamic simulations which can incorporate anharmonic interactions to all orders are restricted to high temperatures or at weak interactions [27]-[29]. Quantum heat transfer studies have been done by Lian Ao Wu et al. by developing a Born-Oppenheimer formalism and have been found that the vibrational or electronic energy dynamics could be described in a new way by the incorporation of quantum effects and nonlinearities [30]. However analytical studies on quantum heat conduction in nonlinear lattices have not been reported yet in the literature. This has prompted us to investigate theoretically the dynamics of heat transfer in nonlinear lattices using the principles of quantum mechanics.

The nonlinear lattices play a vital role in the construction of electronic devices and optical fiber communication systems. Hence we give special consideration to it. The volumetric rise in temperature must be evacuated very efficiently because the heat that is formed by joule effect in these devices will soon reach a level of high temperature and can cause possible failures of the systems. Studies based on temperature and heat effects have been studied by many scientists [31]-[35]. A better understanding of the dynamics of heat conduction may also lead to potentially interesting applications based on the possibility to control the heat flow. Since a sharp knowledge of the phenomena governing the heat transfer must be gained in order to achieve this task, we perform deep investigations on heat conduction in nonlinear lattices. The presence of impurities, defects or imperfections in fact gives rise to inhomogenities and in turn influences the propagation of heat. Hence both homogeneous and inhomogeneous nonlinear lattices have been taken into account for our study.

Hence our overriding concern is with the role of nonlinearity in the conduction of heat in both homogeneous and inhomogeneous lattices which lead to the emergence of stable localized structures. We adopt a different approach namely the standard approach in quantum field theory in order to study the quantum mechanical effects. The main aim of this approach is to follow the method of coherent structures first, i.e. to calculate the Heisenberg equations of motion for the second quantized operators which are required to obey commutation relations. By proposing a model Hamiltonian using the second quantized operators of quantum field theory and averaging it using a suitable wave function, we derive the equations for the field amplitude. The resulting nonlinear equation in its natural form is very difficult to solve because of its high nonlinearity and discreteness. Hence we analyze it numerically and in addition we go for the continuum limit and using the perturbation techniques, we solve the resulting dynamics and analyze the nature of heat conduction in lattices.

Section 2 deals with the numerical analysis of heat conduction in the homogeneous and inhomogeneous nonlinear lattices and Section 3 describes the heat transfer in the continuum level and the results are concluded in Section 4.

\section{Nonlinear Lattice}

\subsection{Homogeneous}

To describe the nonlinear excitations which are ubiquitous in nature and play an important role in the description of a vast variety of natural phenomena, we consider a homogeneous nonlinear lattice consisting of $N$ atoms of equal mass $\mathrm{m}$ and lattice constant $L$. The nonlinear lattice Hamiltonian is described by [36]-[38]

$$
H=\sum_{n=1}^{N} \frac{p_{n}^{2}}{2 m}+U\left(x_{n}\right)+\sum_{n=0}^{N} V\left(x_{n+1}, x_{n}\right)
$$

where $x_{n}$ and $p_{n}$ are the displacements and momenta of the particles about their equilibrium postions. $U\left(x_{n}\right)$ is the on-site potential term. The interaction between nearest neighbors with quartic interparticle interaction potential strength is described by $V\left(x_{n+1}, x_{n}\right)$ which assumes the following form

$$
\begin{gathered}
U\left(x_{n}\right)=\frac{k^{\prime}}{3}\left(x_{n}\right)^{3} \\
V\left(x_{n+1}, x_{n}\right)=\left[\frac{k}{2}\left(x_{n+1}-x_{n}-L\right)^{2}+\frac{\beta}{4}\left(x_{n+1}-x_{n}-L\right)^{4}\right]
\end{gathered}
$$

where $k^{\prime}, k$ and $\beta$ represents the cubic on-site, squared interparticle interaction and quartic interparticle interaction potential strengths respectively. To bring out the nonlinearity, we now make a transition to a second quantized formalism and hence we bosonize the Hamiltonian (1) using the relations 


$$
\begin{gathered}
p_{n}=i \sqrt{\frac{m \hbar \omega}{2}}\left(B_{n}^{\dagger}-B_{n}\right) \\
x_{n}=\rho\left(B_{n}+B_{n}^{\dagger}\right)
\end{gathered}
$$

where $\omega$ is the angular frequency and $\rho=\sqrt{\frac{\hbar}{2 m \omega}}$. The bosonic operators $B_{n}$ and $B_{n}^{\dagger}$ satisfy the usual commutation relations $\left[B_{m}, B_{n}^{\dagger}\right]=\delta_{m, n},\left[B_{m}, B_{n}\right]=\left[B_{m}^{\dagger}, B_{n}^{\dagger}\right]=0$. Using Equation (4) and Equation (5), the Hamiltonian (1) can be written as

$$
\begin{aligned}
H= & \sum_{n=1}^{N} \frac{-\hbar \omega}{4}\left(B_{n}^{\dagger}-B_{n}\right)^{2}+\frac{k^{\prime} \rho^{3}}{3}\left(B_{n}+B_{n}^{\dagger}\right)^{3}+\sum_{n=0}^{N} \frac{k}{2}\left[\delta_{n}\left(\rho^{2} \delta_{n}-L \tau\right)+L^{2}\right] \\
& +\sum_{n=0}^{N} \frac{\beta}{4}\left[\left(\rho \delta_{n}\right)^{4}-4 L\left(\rho \delta_{n}\right)^{3}+6\left(\rho L \delta_{n}\right)^{2}-4 L^{3} \rho \delta_{n}+L^{4}\right] .
\end{aligned}
$$

where $\delta_{n}=B_{n+1}+B_{n+1}^{*}-\left(B_{n}+B_{n}^{*}\right)$ and $\tau=\sqrt{\frac{2 \hbar}{m \omega}}$. In particular we are concerned with nonlinear excitations of atoms due to nonlinearity in the system in which a cluster of atoms may undergo a large excursion as compared to the rest of the atoms. Physically the quantum state of such large amplitude collective modes may be represented by coherent states. Hence we introduce the coherent state representation [39] of a harmonic oscillator given by

$$
|\alpha\rangle=\exp \left(-\frac{|\alpha|^{2}}{2}\right) \sum_{j=0}^{\infty} \frac{\alpha^{j}}{\sqrt{j} !}|j\rangle
$$

We now construct the equation of motion for the boson operator using

$$
i \hbar \frac{\mathrm{d}}{\mathrm{d} t}\left\langle B_{n}\right\rangle=\left\langle\left[B_{n}, H\right]\right\rangle
$$

and

$$
i \hbar \frac{\mathrm{d}}{\mathrm{d} t}\left\langle B_{n}^{\dagger}\right\rangle=\left\langle\left[B_{n}^{\dagger}, H\right]\right\rangle
$$

For the bosonic operators, $B_{n}^{\dagger}|\alpha\rangle=a_{n}^{*}|\alpha\rangle, B_{n}|\alpha\rangle=a_{n}|\alpha\rangle$ with $\langle\alpha \mid \alpha\rangle=1$, where $a_{n}$ is the coherent amplitude of the operator $B_{n}$ for the system in the state $|\alpha\rangle$. Now we write down the equation of motion using Equations (6)-(9) as

$$
\begin{aligned}
i \hbar \frac{\mathrm{d} a_{n}}{\mathrm{~d} t}= & \frac{-\hbar \omega}{2}\left(a_{n}^{*}-a_{n}\right)+k^{\prime} \rho^{3} q_{n}^{2}+k \rho^{2}\left(2 q_{n}-r_{n}-s_{n}\right)+\frac{k L^{2}}{2} \\
& +\beta \rho^{4}\left(2 q_{n}^{3}-r_{n}^{3}-s_{n}^{3}-3 q_{n}^{2} r_{n}+3 r_{n}^{2} q_{n}+3 s_{n}^{2} q_{n}-3 q_{n}^{2} s_{n}\right) \\
& -3 L \beta \rho^{3}\left(r_{n}^{2}-s_{n}^{2}-2 q_{n} r_{n}+2 q_{n} s_{n}\right)+3 L^{2} \beta \rho^{2}\left(2 q_{n}-r_{n}-s_{n}\right)+\frac{L^{4} \beta}{4},
\end{aligned}
$$

Equation (10) represents the nature of heat conduction in the homogeneous nonlinear lattice.

\subsection{Inhomogeneous}

The effect of inhomogenities in the nonlinear lattice during the propagation of heat can be analyzed by the inclusion of site-dependent inhomogenities $f_{n}$ and $g_{n}$ in the Hamiltonian (1) and the inter particle interaction potential term $V\left(x_{n+1}, x_{n}\right)$ is given by

$$
V\left(x_{n+1}, x_{n}\right)=\left[\frac{k}{2} f_{n}\left(x_{n+1}-x_{n}-L\right)^{2}+\frac{\beta}{4} g_{n}\left(x_{n+1}-x_{n}-L\right)^{4}\right]
$$

Using the momentum and positon operators in the corresponding Hamiltonian we get 


$$
\begin{aligned}
H= & \sum_{n=1}^{N} \frac{-\hbar \omega}{4}\left(B_{n}^{\dagger}-B_{n}\right)^{2}+\frac{k^{\prime}}{3} \rho^{3}\left(B_{n}+B_{n}^{?}\right)^{3}+\sum_{n=0}^{N} \frac{k}{2}\left[\rho f_{n} \delta_{n}-L \tau f_{n} \delta_{n}+L\right] \\
& +\sum_{n=0}^{N} \frac{\beta}{4}\left[g_{n}\left(\rho \delta_{n}\right)^{4}-4 L \rho^{3} g_{n} \delta_{n}^{3}+6 L^{2} \rho^{2} g_{n} \delta_{n}^{2}-4 L^{3} \rho g_{n} \delta_{n}+L^{4}\right] .
\end{aligned}
$$

The following equation is derived by the process of commutation method which is solved numerically

$$
\begin{aligned}
i \hbar \frac{\mathrm{d} a_{n}}{\mathrm{~d} t}= & \frac{-\hbar \omega}{2}\left(a_{n}^{*}-a_{n}\right)+k^{\prime} \rho^{3} q_{n}^{2}+\frac{k}{2}\left[\frac{\hbar}{m \omega}\left(f_{n-1} z_{n}+f_{n} y_{n}\right)+L \tau\left(f_{n}-f_{n-1}\right)+L^{2}\right] \\
& +\frac{\beta}{4}\left[\frac{\hbar^{2}}{m^{2} \omega^{2}}\left(g_{n-1} z_{n}^{3}+g_{n} y_{n}^{3}\right)-12 L \rho^{3}\left(g_{n-1} z_{n}^{2}-g_{n} y_{n}^{2}\right)-4 L^{3} \rho\left(g_{n-1}-g_{n}\right)+12 L^{2} \rho^{2}\left(g_{n-1} z_{n}+g_{n} y_{n}\right)+L^{4}\right],
\end{aligned}
$$

where $y_{n}=q_{n}-r_{n}$ and $z_{n}=q_{n}-s_{n}$. Equation (13) is a the discrete equation which describe the nature of heat conduction in the inhomogeneous nonlinear lattice. The periodic inhomogenities are prescribed values as $f_{n}=g_{n}=\tanh (n * h)$. An illustration of the picture displayed in Figure 1 shows that the exponential decay gradually becomes less evident in process of time in the homogeneous nonlinear lattice. Also we observe that the thermal stability is considerably enhanced in the presence of inhomogenities. It is found that the amplitude decreases exponentially in the absence of inhomogenities and the effect of inhomogenities on the propagating solitons is to maintain its amplitude.

\section{Continuum Level}

\subsection{Homogeneous Nonlinear Lattice}

The direct and effective algebraic method for finding exact solutions of nonlinear equations is the tanh method [40]. The function $a_{n}$ changes smoothly over one end of the lattice and hence we can replace $a_{n}(t)$ by $a(x, t)$ and proceed to the continuum limit. Using the Taylor series expansion

$$
a_{n \pm 1}=1 \pm h a_{x}+\frac{h^{2} a_{x x}}{2 !} \pm \frac{h^{3} a_{x x x}}{3 !}+\frac{h^{4} a_{x x x x}}{4 !} \pm \cdots
$$

where $h$ is the lattice parameter. Using equation (14), equation (13) takes the form
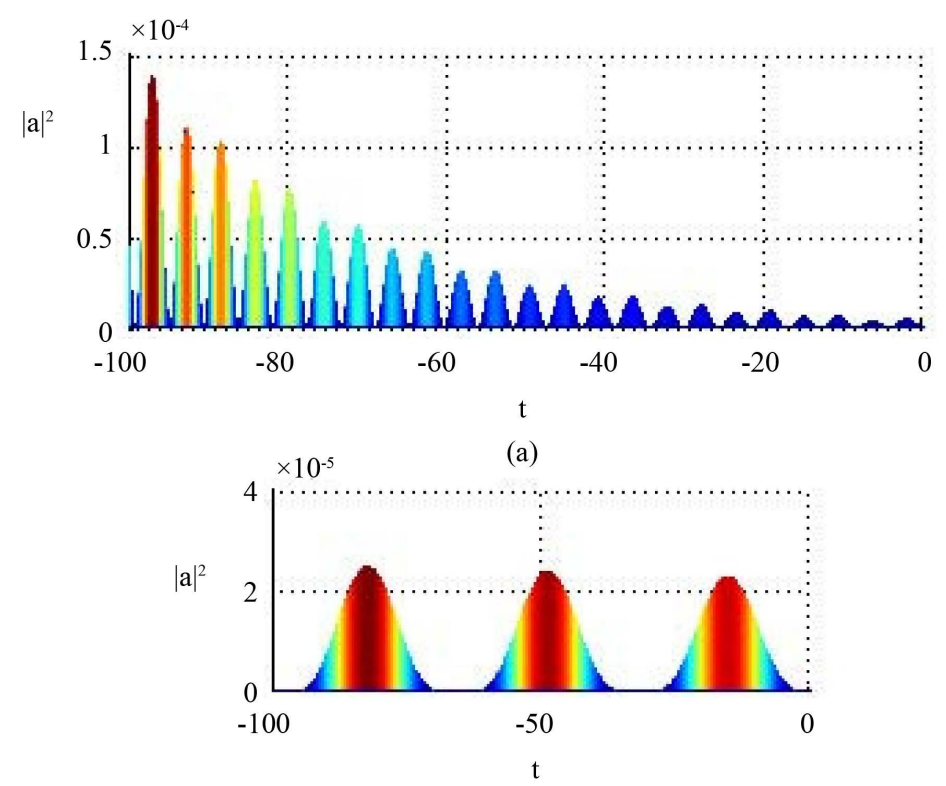

(b)

Figure 1. Snapshot of solitons in the (a) homogeneous nonlinear lattice (b) inhomogeneous nonlinear lattice. 


$$
\begin{aligned}
i \hbar \frac{\mathrm{d} a}{\mathrm{~d} t}= & -\frac{\hbar \omega}{2}\left(a^{*}-a\right)+k^{\prime} \rho^{3} \omega^{2}-\frac{4 k \pi^{2} \hbar^{3}}{m \omega}\left(\frac{1}{2 !} w_{x x}+\frac{h^{2}}{4 !} w_{x x x x}\right)+\frac{k L^{2}}{2}-\frac{12 \hbar^{6} \beta \pi^{4}}{m^{2} \omega^{2}} w_{x x}\left(w_{x}^{2}+2 a_{x}^{*} a_{x}\right) \\
& +6 L h^{3} \beta \rho^{3}\left(w_{x} w_{x x}\right)-6 L^{2} h^{2} \beta \rho\left(\frac{1}{2} w_{x x}+\frac{1}{4 !} w_{x x x x}\right)+\frac{L^{4} \beta}{4},
\end{aligned}
$$

where, $w=a+a^{*}$. Using $a=U+i V$, the Equation (15) is reduced to the following crucial step by substituting the imaginary part into the real one

$$
A U_{t t}+C U^{2}-E U_{x x}-F U_{x x x x}+G-H U_{x x}-I U_{x x x x}-J U_{x}^{2} U_{x x}+K U_{x} U_{x x}+P=0
$$

where

$$
\begin{aligned}
& A=\frac{\hbar}{\omega}, \quad C=\rho 4 k^{\prime}, \quad E=\frac{4 k \pi^{2} \hbar^{3}}{m \omega}, \quad F=\frac{k h^{2} \hbar^{3} \pi^{2}}{3 m \omega}, \quad G=\frac{k L^{2}}{2}, \\
& H=6 L^{2} h^{2} \beta \rho, \quad I=\frac{L^{2} h^{4} \beta}{2}, \quad J=\frac{96 \hbar^{6} \beta \pi^{4}}{m^{2} \omega^{2}}, \quad K=24 L^{2} h^{3} \beta \rho^{3}, \quad P=\frac{L^{4} \beta}{4} .
\end{aligned}
$$

Equation (16) is a nonintegrable equation which can only be solved using perturbation techniques and for the present study we employ the tanh method which leads to a further systemization of the method and seek solutions in the travelling frame of reference [40]

$$
\xi=\sum_{j=1}^{N}\left(c_{j} x_{j}\right)
$$

where the components $c_{j}$ of the wave vector $\mathrm{x}$ are constants. We seek polynomial solutions expressible in hyperbolic tangent $T=\tanh (\xi)$. Based on the identity $\cosh ^{2}(\xi)-\sinh ^{2}(\xi)=1$,

$$
\begin{gathered}
\tanh ^{\prime}(\xi)=\operatorname{sech}^{2}(\xi)=1-\tanh ^{2}(\xi) \\
\tanh ^{\prime \prime}(\xi)=-2 \tanh (\xi)+2 \tanh ^{3}(\xi), \text { etc. }
\end{gathered}
$$

Therefore the first and consequently all higher order derivatives are polynomial in $T$. Thus repeatedly applying the chain rule

$$
\frac{\partial \bullet}{\partial x_{j}}=\frac{\partial \xi}{\partial x_{j}} \frac{\mathrm{d} T}{\mathrm{~d} \xi} \frac{\mathrm{d} \bullet}{\mathrm{d} T}=c_{j}\left(1-T^{2}\right) \frac{\mathrm{d} \bullet}{\mathrm{d} T}
$$

Equation (16) can be transformed into a coupled system of nonlinear ODEs

$$
\Gamma\left(T, U(T), U^{\prime}(T), \cdots\right)=0
$$

where $U_{n}(T)$ corresponds to $a_{n}(x)$. Using this technique, Equation (16) is reduced into a nonlinear ODE as follows

$$
\begin{aligned}
& A c_{2}^{2}\left[U^{\prime} \phi_{2}+U^{\prime \prime} \phi_{1}\right]+C U^{2}-E c_{1}^{2}\left[U^{\prime} \phi_{2}+U^{\prime \prime} \phi_{1}\right]-F c_{1}^{4}\left[U^{\prime} \phi_{3}+U^{\prime \prime} \phi_{4}+U^{\prime \prime \prime} \phi_{5}+U^{\prime \prime \prime \prime} \phi_{6}\right]-H c_{1}^{2}\left[U^{\prime} \phi_{2}+U^{\prime \prime} \phi_{1}\right] \\
& -I c_{1}^{4}\left[U^{\prime} \phi_{3}+U^{\prime \prime} \phi_{4}+U^{\prime \prime \prime} \phi_{5}+U^{\prime \prime \prime \prime} \phi_{6}\right]-J c_{1}^{4}\left[U^{\prime} U^{\prime 2} \phi_{7}+U^{\prime \prime} U^{\prime 2} \phi_{6}\right]+K c_{1}^{3}\left[U^{\prime} U^{\prime \prime} \phi_{8}+U^{\prime 2}\left(\phi_{9}\right)\right]+P+G=0 .
\end{aligned}
$$

where

$$
\begin{aligned}
& \phi_{1}=1-2 T^{2}+T^{4}, \quad \phi_{2}=-2 T+2 T^{3}, \quad \phi_{3}=16 T-40 T^{3}+24 T^{5}, \quad \phi_{4}=-8+52 T^{2}-80 T^{4}+36 T^{6}, \\
& \phi_{5}=-12 T+36 T^{3}-36 T^{5}+12 T^{7}, \quad \phi_{6}=1-4 T^{2}+6 T^{4}-4 T^{6}+T^{8}, \quad \phi_{7}=-2 T+6 T^{3}-6 T^{5}+2 T^{7}, \\
& \phi_{8}=1-3 T^{2}+3 T^{4}-T^{6}, \quad \phi_{9}=-2 T+4 T^{3}-2 T^{5} .
\end{aligned}
$$

The tanh method admits the use of the finite expansion

$$
U(T)=\sum_{j=0}^{M}\left(a_{j} T^{j}\right)
$$

By balancing the linear and nonlinear terms in $T$, the value of $M$ is found to be equal to 1 . Equating the coefficients of the powers of $T$, we get 


$$
\begin{gathered}
G+P+C a_{0}^{2}=0 \\
-2 c_{2}^{2} a_{1} A+2 E c_{1}^{2} a_{1}-16 F c_{1}^{4} a_{1}+2 H c_{1}^{2} a_{1}-16 I c_{1}^{4} a_{1}+2 J c_{1}^{4} a_{1}^{3}-2 K c_{1}^{3} a_{1}^{2}+2 C a_{0} a_{1}=0 \\
C a_{1}^{2}=0 \\
2 A c_{2}^{2} a_{1}-2 E c_{1}^{2} a_{1}+40 F c_{1}^{4} a_{1}-2 H c_{1}^{2} a_{1}+40 I c_{1}^{4} a_{1}-6 J c_{1}^{4} a_{1}^{3}+4 K c_{1}^{3} a_{1}^{2}=0 \\
-24 F c_{1}^{4} a_{1}-24 I c_{1}^{4} a_{1}+6 J c_{1}^{4} a_{1}^{3}-2 K c_{1}^{3} a_{1}^{2}=0 \\
-2 J c_{1}^{4} a_{1}^{3}=0
\end{gathered}
$$

Assuming $J=0$ and $a_{1} \neq 0$ from the equations we get

$$
a_{1}=\frac{-2 A c_{2}^{2}+2 E c_{1}^{2}-40 F c_{1}^{4}+2 H c_{1}^{2}-40 I c_{1}^{4}}{4 K c_{1}^{3}}
$$

The remaining constants G,A and $\mathrm{K}$ are now easily found through simple algebra by applying the conditions. As a result we get,

$$
\begin{gathered}
G=-P \\
A=\frac{E c_{1}^{2}-8 F c_{1}^{4}+H c_{1}^{2}-8 I c_{1}^{4}-K a_{1} c_{1}^{3}}{c_{2}^{2}} \\
K=\frac{-12 F c_{1}-12 I c_{1}}{a_{1}}
\end{gathered}
$$

By solving the equations we obtain the explicit solution

$$
U=\frac{-2 A c_{2}^{2}+2 E c_{1}^{2}-40 F c_{1}^{4}+2 H c_{1}^{2}-40 I c_{1}^{4}}{4 K c_{1}^{3}} \tanh \left(c_{1} x+c_{2} t\right)
$$

The propagation of solitons in the homogeneous nonlinear lattice, preserving their phase and shape is given by the solution (36).

\subsection{Inhomogeneous Nonlinear Lattice}

To note the propagation of solitons in the presence of inhomogenities we use the prescribed values as $f_{n}=g_{n}=\sin (n)$ and the application of continuous approximation process to Equation (13) generates,

$i \hbar \frac{\mathrm{d} a}{\mathrm{~d} t}$

$$
\begin{aligned}
= & \alpha_{1}\left(a^{*}-a\right)+k^{\prime} \rho^{3} \omega^{2} \\
& +\alpha_{2}\left(-2 \mathrm{e}^{2} w_{x x}-2 f_{x} \mathrm{e}^{2} w_{x}+f_{x} \mathrm{e}^{3} w_{x x}+\mathrm{e}^{3} f_{x x} w_{x}-\frac{1}{2} f_{x x} \mathrm{e}^{4} w_{x x}-\frac{1}{3} f_{x x x} \mathrm{e}^{4} w_{x}-\frac{1}{3} f_{x} \mathrm{e}^{4} w_{x x x}-\frac{1}{6} f \mathrm{e}^{4} w_{x x x x}\right) \\
& +\alpha_{3}\left(f_{x} \mathrm{e}-\frac{1}{2} f_{x x} \mathrm{e}^{2}+\frac{1}{6} f_{x x x} \mathrm{e}^{3}-\frac{1}{24} f_{x x x x} \mathrm{e}^{4}\right)+\frac{k L^{2}}{2}+\alpha_{4}\left(-3 a_{x}^{2} g \mathrm{e}^{4} w_{x x}-g_{x} \mathrm{e}^{4} w_{x}^{3}-6 a_{x} g \mathrm{e}^{4} a_{x}^{*} w_{x x}-3 g \mathrm{e}^{4} a_{x}^{* 2} w_{x x}\right) \\
& +\alpha_{5}\left(-g_{x} \mathrm{e}^{3} w_{x}^{2}-2 a_{x x}^{*} g \mathrm{e}^{3} w_{x}-2 a_{x x} g \mathrm{e}^{3} w_{x}+a_{x x} g_{x} \mathrm{e}^{4} w_{x}+\frac{1}{2} g_{x x} \mathrm{e}^{4} w_{x}^{2}+g_{x} \mathrm{e}^{4} a_{x x}^{*} w_{x}\right) \\
& +\alpha_{6}\left(-g_{x} \mathrm{e}+\frac{1}{2} g_{x x} \mathrm{e}^{2}-\frac{1}{6} g_{x x x} \mathrm{e}^{3}+\frac{1}{24} g_{x x x} \mathrm{e}^{4}\right)+\alpha_{7}\left[\left(4 w g-2 w g_{x} \mathrm{e}+w g_{x x} \mathrm{e}^{2}-\frac{1}{3} g_{x x x} \mathrm{e}^{3} w+\frac{1}{12} g_{x x x x} \mathrm{e}^{4} w\right)\right. \\
& -2\left(2 g w-g_{x} \mathrm{e}+g \mathrm{e}^{2} w_{x x}+\frac{1}{2} w g_{x x} \mathrm{e}^{2}+g_{x} \mathrm{e}^{2} w_{x}-\frac{1}{2} g_{x} \mathrm{e}^{3} w_{x x}-\frac{1}{2} g_{x x} \mathrm{e}^{3} w_{x}-\frac{1}{6} w g_{x x x} \mathrm{e}^{3}+\frac{1}{12} g \mathrm{e}^{4} w_{x x x x}+\frac{1}{6} g_{x} \mathrm{e}^{4} w_{x x x}\right. \\
& \left.\left.+\frac{1}{4} g_{x x} \mathrm{e}^{4} w_{x x}+\frac{1}{6} g_{x x x} \mathrm{e}^{4} w_{x}+\frac{1}{24} g_{x x x x} \mathrm{e}^{4} w_{x}\right)\right]+\frac{L^{4} \beta}{4},
\end{aligned}
$$


where

$$
\begin{gathered}
\alpha_{1}=\frac{-\hbar \omega}{2}, \quad \alpha_{2}=\frac{k \rho^{2}}{2}, \quad \alpha_{3}=\frac{k L}{2} \tau, \quad \alpha_{4}=\beta \rho^{4}, \\
\alpha_{5}=-3 L \beta \rho^{3}, \quad \alpha_{6}=-L^{3} \beta \rho, \quad \alpha_{7}=\frac{3 L^{2} \beta}{2} \rho,
\end{gathered}
$$

and e is the lattice parameter. Equation (37) can be consequently reduced to the following form by using $a=U+i V$ and substituting the imaginary part into the real part, we get

$$
\begin{aligned}
& K_{1} U_{t t}+K_{2} U+s_{1} U_{x}+s_{2} U_{x x}-s_{3} U_{x x x}-s_{4} U_{x x x x}+K_{11} U_{x} U_{x x}-K_{12} U_{x x x} U_{x}-K_{9} U_{x}^{2} U_{x x}+K_{10} U_{x}^{2}-K_{8} U_{x}^{3} \\
& \quad+K_{7}+K_{17}=0 .
\end{aligned}
$$

where

$$
\begin{gathered}
K_{1}=\frac{\hbar}{\omega}, \quad K_{2}=\frac{k^{\prime} \hbar}{m \omega}, \quad K_{3}=\frac{k \mathrm{e}^{4} f_{x} \hbar}{6 m \omega}, \\
K_{4}=-\frac{k \mathrm{e}^{2} f_{x} \hbar}{m \omega}+\frac{k \mathrm{e}^{3} f_{x x} \hbar}{2 m \omega}-\frac{k \mathrm{e}^{4} f_{x x x} \hbar}{6 m \omega} \\
K_{5}=-\frac{k \mathrm{e}^{2} f \hbar}{m \omega}+\frac{k \mathrm{e}^{3} f_{x} \hbar}{2 m \omega}-\frac{k \mathrm{e}^{4} f_{x x} \hbar}{4 m \omega}, \quad K_{6}=\frac{k \mathrm{e}^{4} f \hbar}{12 m \omega} \\
K_{7}=\frac{k L \mathrm{e} f_{x} \hbar}{4 m \omega} \tau-\frac{k L \mathrm{e}^{2} f_{x x} \hbar}{8 m \omega} \tau+\frac{k L \mathrm{e}^{3} f_{x x x} \hbar}{24 m \omega} \tau-\frac{k L \mathrm{e}^{4} f_{x x x x} \hbar}{96 m \omega} \tau+\frac{k L^{3} \hbar}{4 m \omega} \tau, \quad K_{8}=\frac{2 \mathrm{e}^{4} g_{x} \beta \hbar^{2}}{m^{2} \omega^{2}}, \quad K_{9}=\frac{6 \mathrm{e}^{4} g \beta \hbar^{2}}{m^{2} \omega^{2}} \\
K_{10}=12 L \mathrm{e}^{3} g_{x} \beta \rho^{3}-6 \mathrm{e}^{4} L g_{x x} \beta \rho^{3}, \quad K_{11}=24 L \mathrm{e}^{3} g \beta \rho^{3} \\
K_{12}=12 L \mathrm{e}^{4} g_{x} \beta \rho^{3}, \quad K_{13}=L^{2} \mathrm{e}^{4} g_{x} \beta \rho \\
K_{14}=-6 \mathrm{e}^{2} L^{2} g_{x} \beta \rho+3 \mathrm{e}^{3} L^{2} g_{x x} \beta \rho-\mathrm{e}^{4} L^{2} g_{x x x} \beta \rho \\
K_{15}=-6 L^{2} \mathrm{e}^{2} g \beta \rho+3 L^{2} \mathrm{e}^{3} g_{x} \beta \rho-\frac{3 L^{2} \mathrm{e}^{4} g_{x x} \beta}{2} \rho, \quad K_{16}=\frac{L^{2} \mathrm{e}^{4} g \beta}{2} \rho \\
K_{17}=L^{3} \mathrm{e}_{x} \beta \rho-\frac{L^{3} \mathrm{e}^{2} g_{x x} \beta}{2} \rho+\frac{L^{3} \mathrm{e}^{3} g_{x x x} \beta}{6} \rho-\frac{L^{3} \mathrm{e}^{4} g_{x x x x} \beta}{24} \rho+\frac{L^{4} \beta}{4} \\
S_{1}=K_{4}+K_{14}, \quad s_{2}=K_{5}+K_{15}, \quad s_{3}=K_{3}+K_{13}, \quad s_{4}=K_{6}+K_{16}
\end{gathered}
$$

The transformation of Equation (40) to a nonlinear ODE is possible by the repeated application of chain rule (21) which is given by

$$
\begin{aligned}
& K_{1} c_{2}^{2}\left[U^{\prime} \phi_{2}+U^{\prime \prime} \phi_{1}\right]+K_{2}-K_{3} c_{1}^{3}\left[U^{\prime} \phi_{10}+U^{\prime \prime} \phi_{11}+U^{\prime \prime \prime} \phi_{8}\right]+K_{4} c_{1} U^{\prime} \phi_{12}+K_{5} c_{1}^{2}\left[U^{\prime} \phi_{2}+U^{\prime \prime} \phi_{1}\right] \\
& -K_{6} c_{1}^{4}\left[U^{\prime} \phi_{3}+U^{\prime \prime} \phi_{4}+U^{\prime \prime \prime} \phi_{5}+U^{\prime \prime \prime \prime} \phi_{6}\right]-K_{8} c_{1}^{3}\left[U^{\prime 3} \phi_{8}\right]-K_{9} c_{1}^{4}\left[U^{\prime 2} U^{\prime} \phi_{7}+U^{\prime 2} U^{\prime \prime} \phi_{6}\right]+K_{10} c_{1}^{2} U^{\prime 2} \phi_{1} \\
& +K_{11} c_{1}^{3}\left[U^{\prime} U^{\prime \prime} \phi_{8}+U^{\prime 2} \phi_{9}\right]-K_{12} c_{1}^{4}\left[U^{\prime \prime} U^{\prime}\left(-6 T+18 T^{3}-18 T^{5}+6 T^{7}\right)+U^{\prime} U^{\prime \prime \prime} \phi_{6}+U^{\prime 2}\left(-2+10 T^{2}-14 T^{4}+6 T^{6}\right)\right] \\
& -K_{13} c_{1}^{3}\left[U^{\prime} \phi_{10}+U^{\prime \prime} \phi_{11}+U^{\prime \prime \prime} \phi_{8}\right]+K_{14} c_{1} U^{\prime} \phi_{12}+K_{15} c_{1}^{2}\left[U^{\prime} \phi_{2}+U^{\prime \prime} \phi_{1}\right]-K_{16} c_{1}^{4}\left[U^{\prime} \phi_{3}+U^{\prime \prime} \phi_{4}+U^{\prime \prime \prime} \phi_{5}+U^{\prime \prime \prime \prime} \phi_{6}\right] \\
& +K_{7}+K_{17}=0 .
\end{aligned}
$$

where

$$
\phi_{10}=-2+8 T^{2}-6 T^{4}, \quad \phi_{11}=-6 T+12 T^{3}-6 T^{5}, \quad \phi_{12}=1-T^{2}
$$

Balancing the linear and nonlinear terms we get $M=1$. Equating the coefficients of the highest powers of $T$ we get

$$
K_{7}+K_{17}-K_{8} c_{1}^{3} a_{1}^{3}+2 K_{12} c_{1}^{4} a_{1}^{2}+K_{10} c_{1}^{2} a_{1}^{2}+K_{4} c_{1} a_{1}+K_{14} c_{1} a_{1}+2 K_{3} c_{1}^{3} a_{1}+2 K_{13} c_{1}^{3} a_{1}+K_{2} a_{0}^{2}=0
$$




$$
\begin{gathered}
-16 K_{6} c_{1}^{4} a_{1}-2 K_{5} c_{1}^{2} a_{1}-16 K_{16} c_{1}^{4} a_{1}-2 K_{15} c_{1}^{2} a_{1}+2 K_{9} c_{1}^{4} a_{1}^{3}-2 K_{11} c_{1}^{3} a_{1}^{2}-2 K_{1} c_{2}^{2} a_{1}+2 K_{2} a_{0} a_{1}=0 \\
-K_{4} c_{1} a_{1}-K_{14} c_{1} a_{1}-8 K_{3} c_{1}^{3} a_{1}-8 K_{13} c_{1}^{3} a_{1}+3 K_{8} c_{1}^{3} a_{1}^{3}-10 K_{12} c_{1}^{4} a_{1}^{2}-2 K_{10} c_{1}^{2} a_{1}^{2}+K_{2} a_{1}^{2}=0 \\
2 K_{1} c_{2}^{2} a_{1}+40 K_{6} c_{1}^{4} a_{1}+2 K_{5} c_{1}^{2} a_{1}+40 K_{16} c_{1}^{4} a_{1}+2 K_{15} c_{1}^{2} a_{1}-6 K_{9} c_{1}^{4} a_{1}^{3}+4 K_{11} c_{1}^{3} a_{1}^{2}=0 \\
6 K_{3} c_{1}^{3} a_{1}+6 K_{13} c_{1}^{3} a_{1}-3 K_{8} c_{1}^{3} a_{1}^{3}+14 K_{12} c_{1}^{4} a_{1}^{2}+K_{10} c_{1}^{2} a_{1}^{2}=0 \\
-24 K_{6} c_{1}^{4} a_{1}-24 K_{16} c_{1}^{4} a_{1}+6 K_{9} c_{1}^{4} a_{1}^{3}-2 K_{11} c_{1}^{3} a_{1}^{2}=0 \\
K_{8} c_{1}^{3} a_{1}^{3}-6 K_{12} c_{1}^{4} a_{1}^{2}=0 \\
-2 K_{9} c_{1}^{4} a_{1}^{3}=0
\end{gathered}
$$

Assuming $K_{9}=0$ and $a_{1} \neq 0$ from the above equations,

$$
\begin{gathered}
a_{0}=\frac{16 c_{1}^{4} K_{6} K_{8}+2 c_{1}^{2} K_{5} K_{8}+16 c_{1}^{4} K_{16} K_{8}}{2 K_{2} K_{8}}+\frac{2 c_{1}^{2} K_{15} K_{8}+12 c_{1}^{4} K_{11} K_{12}+2 K_{1} c_{2}^{2} K_{8}}{2 K_{2} K_{8}} \\
a_{1}=\frac{6 K_{12} c_{1}}{K_{8}}
\end{gathered}
$$

and adhering to the conditions we get

$$
\begin{gathered}
K_{7}=-K_{2} a_{0}^{2}-K_{17}+K_{8} c_{1}^{3} a_{1}^{3}-2 K_{12} c_{1}^{4} a_{1}^{2}-K_{10} c_{1}^{2} a_{1}^{2}-K_{4} c_{1} a_{1}-K_{14} c_{1} a_{1}-2 K_{3} c_{1}^{3} a_{1}-2 K_{13} c_{1}^{3} a_{1} \\
K_{4}=\frac{K_{2} a_{1}^{2}-2 c_{1}^{2} a_{1}^{2} K_{10}-c_{1} K_{14} a_{1}-8 c_{1}^{3} K_{3} a_{1}}{c_{1} a_{1}} \frac{-8 c_{1}^{3} K_{13} a_{1}+3 c_{1}^{3} K_{8} a_{1}^{3}-10 c_{1}^{4} K_{12} a_{1}^{2}}{c_{1} a_{1}} \\
K_{1}=\frac{-40 K_{6} c_{1}^{4}-2 K_{5} c_{1}^{2}-40 K_{16} c_{1}^{4}-2 K_{15} c_{1}^{2}-4 K_{11} c_{1}^{3} a_{1}}{2 c_{2}^{2}} \\
K_{3}=\frac{-6 K_{13} c_{1}+3 K_{8} c_{1} a_{1}^{2}-14 K_{12} c_{1}^{2} a_{1}-K_{10} a_{1}}{6 c_{1}} \\
K_{6}=\frac{-24 K_{16} c_{1}-2 K_{11} a_{1}}{24 c_{1}}
\end{gathered}
$$

The following solution is obtained by solving the Equations from (53) to (60),

$$
U=\frac{16 c_{1}^{4} K_{8} K_{6}+2 c_{1}^{2} K_{5} K_{8}+16 c_{1}^{4} K_{16} K_{8}}{2 K_{2} K_{8}}+\frac{2 c_{1}^{2} K_{15} K_{8}+12 c_{1}^{4} K_{11} K_{12}+2 c_{2}^{2} K_{1} K_{8}}{2 K_{2} K_{8}}+\frac{6 K_{12} c_{1}}{K_{8}} \tanh \left(c_{1} x+c_{2} t\right)
$$

Equation (68) describes the nature of soliton propagation in the inhomogeneous nonlinear lattice. In this context it is noted that the effect of inhomogenities in the form of tangent hyperbolic functions on soliton propagation induces a phase shift. Thus it is worth mentioning that the soliton on interaction with the inhomogenities which arises due to defects or imperfections acquires a phase shift. Hence the only change the wave experiences in the presence of inhomogenities is a shift in their relative phases and is shown in Figure 2.

\section{Conclusions}

To conclude, we summarize the main results. We have investigated the heat transport properties in various models like homogeneous and inhomogeneous nonlinear lattices with cubic on-site potential and quartic interparticle interaction potential, by considering a model Hamiltonian using the second quantized operators of quantum field theory. We derived the equations for the field amplitude and after averaging the Hamiltonian using a suitable wave function, we analyzed it numerically and analytically.

From the numerical results of the homogeneous nonlinear lattice model, it is found that the soliton pro pagation has ultimately resulted in the dissipation of heat in an exponential fashion. On the other hand, the presence of inhomogenities almost maintains the amplitude of the soliton, propagating in the inhomogeneous 
(a)

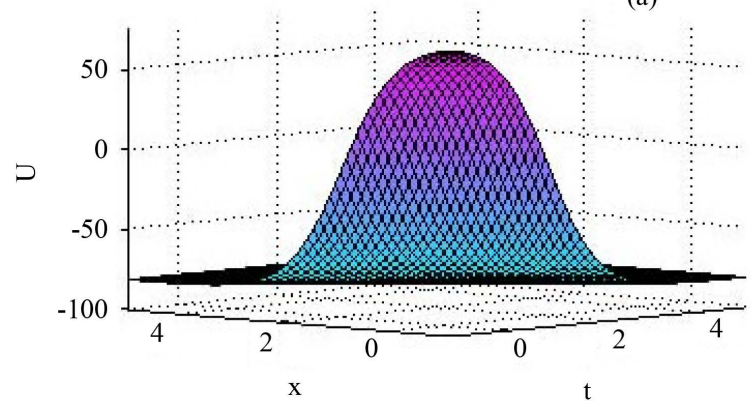

(b)

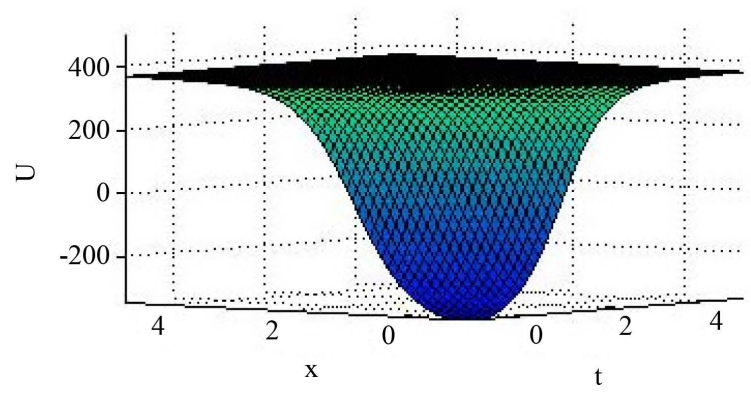

Figure 2. Snapshot of the solitary pulses in the (a) homogeneous (b) inhomogeneous nonlinear lattice in the continuum level.

nonlinear lattice. Hence this lattice is found to support the amplitude of heat without any energy loss and it could be used to design the heat transferring devices to transfer heat without energy loss. The realisation of soliton-like heat dissipation on the nonlinear lattice has clearly demonstrated that the results of the fundamental soliton theory can be successfully exploited in very important practical applications. From the continuum results it is found that the inhomogeneous structure accounts well for the shifting of the phase of the solitons propagating in the inhomogeneous nonlinear lattice. This suggests that the simplicity of this concept could be used in heat transferring devices which transfer heat using phase shift mechanism for instance.

\section{References}

[1] Saito, K. (2003) Strong Evidence of Normal Heat Conduction in a One-Dimensional Quantum System. Europhysics Letters, 61, 34.

[2] Zurcher, U. and Talkner, P. (1900) Quantum-Mechanical Harmonic Chain Attached to Heat Baths. II. Nonequilibrium Properties. Physical Review A, 42, 3278. http://dx.doi.org/10.1103/PhysRevA.42.3278

[3] Roy, D. and Dhar, A. (2008) Role of Pinning Potentials in Heat Transport through Disordered Harmonic Chains. Physical Review E, 78, Article ID: 051112. http://dx.doi.org/10.1103/PhysRevE.78.051112

[4] O’Connor, A.J. and Lebowitz, J.L. (1974) Heat Conduction and Sound Transmission in Isotopically Disordered Harmonic Crystals. Journal of Mathematical Physics, 15, 692. http://dx.doi.org/10.1063/1.1666713

[5] Eckmann, J.P., Pillet, C.A. and Rey-Bellet L. (1999) Non-Equilibrium Statistical Mechanics of Anharmonic Chain Coupled to Two Heat Baths at Different Temperatures. Communications in Mathematical Physics, 201, 657.

[6] Verheggen, T. (1979) Transmission Coefficient and Heat Conduction of a Harmonic Chain with Random Masses: Asymptotic Estimates on Products of Random Matrices. Communications in Mathematical Physics, 68, 69.

[7] Dhar, A. (2001) Comment on “Can Disorder Induce a Finite Thermal Conductivity in 1D Lattices?” Physical Review Letters, 87, Article ID: 069401.

[8] Mejia-Monasterio, C., Larralde, H. and Leyvraz, F. (2001) Coupled Normal Heat and Matter Transport in a Simple Model System. Physical Review Letters, 86, 5417.s

[9] Li, B., Wang, J. and Casati, G. (2003) Heat Conductivity in Linear Mixing Systems. Physical Review E, 67, 021204. http://dx.doi.org/10.1103/PhysRevE.67.021204

[10] Dhar, A. and Roy, D. (2006) Heat Transport in Harmonic Lattices. Journal of Statistical Physics, 125, 801-820. http://dx.doi.org/10.1007/s10955-006-9235-3

[11] Li, B.W., Wang, L. and Hu, B. (2002) Finite Thermal Conductivity in 1D Models Having Zero Lyapunov Exponents. Physical Review Letters, 88, Article ID: 223901. http://dx.doi.org/10.1103/PhysRevLett.88.223901

[12] Nakazawa, H. (1968) Energy Flow in Harmonic Linear Chain. Progress of Theoretical Physics, 39, $236-238$. http://dx.doi.org/10.1143/PTP.39.236

[13] Li, B.W., Zhao, H. and Hu, B. (2001) Can Disorder Induce a Finite Thermal Conductivity in 1D Lattices? Physical Review Letters, 86, 63. http://dx.doi.org/10.1103/PhysRevLett.86.63

[14] Mountain, R.D. and MacDonald, R.A. (1983) Thermal Conductivity of Crystals: A Molecular-Dynamics Study of Heat Flow in a Two-Dimensional Crystal. Physical Review B, 28, 3022. http://dx.doi.org/10.1103/PhysRevB.28.3022

[15] Jackson, E.A. and Mistriotis, A.D. (1989) Thermal Conductivity of One- and Two-Dimensional Lattices. Journal of Physics: Condensed Matter, 1, 1223. http://dx.doi.org/10.1088/0953-8984/1/7/006 
[16] Dhar, A. (2001) Heat Conduction in the Disordered Harmonic Chain Revisited. Physical Review Letters, 86, 5882. http://dx.doi.org/10.1103/PhysRevLett.86.5882

[17] Lepri, S., Livi, R. and Politi, A. (1997) Heat Conduction in Chains of Nonlinear Oscillators. Physical Review Letters, 78, 1896. http://dx.doi.org/10.1103/PhysRevLett.78.1896

[18] Rubin, R.J. and Greer, W.L. (1971) Abnormal Lattice Thermal Conductivity of a One-Dimensional, Harmonic, Isotopically Disordered Crystal. Journal of Mathematical Physics, 12, 1686.

[19] Shiba, H. and Ito, N. (2008) Anomalous Heat Conduction in Three-Dimensional Nonlinear Lattices. Journal of the Physical Society of Japan, 77, Article ID: 054006. http://dx.doi.org/10.1143/JPSJ.77.054006

[20] Bourbonnais, R. and Maynard, R. (1990) Energy Transport in One- and Two-Dimensional Anharmonic Lattices with Isotopic Disorder. Physical Review Letters, 64, 1397. http://dx.doi.org/10.1103/PhysRevLett.64.1397

[21] Terraneo, M., Peyrard, M. and Casati, G. (2002) Controlling the Energy Flow in Nonlinear Lattices: A Model for a Thermal Rectifier. Physical Review Letters, 88, Article ID: 094302. http://dx.doi.org/10.1103/PhysRevLett.88.094302

[22] Li, B.W., Wang, L. and Casati, G. (2006) Negative Differential Thermal Resistance and Thermal Transistor. Applied Physics Letters, 88, Article ID: 143501. http://dx.doi.org/10.1063/1.2191730

[23] Gaul, C. and Büttner, H. (2007) Quantum Mechanical Heat Transport in Disordered Harmonic Chains. Physical Review E, 76, Article ID: 011111. http://dx.doi.org/10.1103/PhysRevE.76.011111

[24] Segal, D. and Nitzan, A. (2005) Spin-Boson Thermal Rectifier. Physical Review Letters, 94, Article ID: 034301. http://dx.doi.org/10.1103/PhysRevLett.94.034301

[25] Li, B.W., Wang, L. and Casati, G. (2004) Thermal Diode: Rectification of Heat Flux. Physical Review Letters, 93, Article ID: 184301. http://dx.doi.org/10.1103/PhysRevLett.93.184301

[26] Toda, M. (1979) Solitons and Heat Conduction. Physica Scripta, 20, 424. http://dx.doi.org/10.1088/0031-8949/20/3-4/017

[27] Dhar, A. (2008) Heat Transport in Low-Dimensional Systems. Advances in Physics, 57, 457-537. http://dx.doi.org/10.1080/00018730802538522

[28] Wang, J.S. (2007) Quantum Thermal Transport from Classical Molecular Dynamics. Physical Review Letters, 99, Article ID: 160601. http://dx.doi.org/10.1103/PhysRevLett.99.160601

[29] Stock, G. (2009) Classical Simulation of Quantum Energy Flow in Biomolecules. Physical Review Letters, 102, Article ID: 118301. http://dx.doi.org/10.1103/PhysRevLett.102.118301

[30] Wu, L.A. and Segal, D. (2011) Quantum Heat Transfer: A Born-Oppenheimer Method. Physical Review E, 83, Article ID: 051114. http://dx.doi.org/10.1103/PhysRevE.83.051114

[31] Imai, H., Wada, H. and Shiga, M. (1995) Effects of Spin Fluctuations on the Specific-Heat in YMN2 and Y0.97SC0.03MN2. Journal of the Physical Society of Japan, 64, 2198.

[32] Theodorakopoulos, N. and Bacalis, N.C. (1992) Thermal Solitons in the Toda Chain. Physical Review B, 46, 10706. http://dx.doi.org/10.1103/PhysRevB.46.10706

[33] Takayama, H. and Ishikawa, M. (1986) Classical Thermodynamics of the Toda Lattice as a Classical Limit of the TwoComponent Bethe Ansatz Scheme. Progress of Theoretical Physics, 76, 820.

[34] Theodorakopoulos, N. (1984) Ideal-Gas Approach to the Statistical Mechanics of Integrable Systems: The Sine-Gordon Case. Physical Review B, 30, 4071. http://dx.doi.org/10.1103/PhysRevB.30.4071

[35] Majernik, V. and Majernikova, E. (1995) The Possibility of Thermal Solitons. International Journal of Heat and Mass Transfer, 38, 2701-2703.

[36] Li, N.B., Zhan, F., Hanggi, P. and Li, B.W. (2009) Shuttling Heat across One-Dimensional Homogenous Nonlinear Lattices with a Brownian Heat Motor. Physical Review E, 80, Article ID: 011125. http://dx.doi.org/10.1103/PhysRevE.80.011125

[37] Dhar, A. (2008) Heat Transport in Low-Dimensional Systems. Advances in Physics, 57, 5.

[38] Stefano, L., Livi, R. and Politi, A. (2003) Thermal Conduction in Classical Low-Dimensional Lattices. Arxiv: Condmat/0112193v2.

[39] Radcliffe, J. M. (1971) Some Properties of Coherent Spin States. Journal of Physics A: General Physics, 4, 313.

[40] Baldwin, D., Goklas, U. and Hereman, W. (2004) Symbolic Computation of Hyperbolic Tangent Solutions for Nonlinear Differential-Difference Equations. Computer Physics Communications, 162, 203-217. http://dx.doi.org/10.1016/j.cpc.2004.07.002 